\title{
NICMOS CORONAGRAPHIC OBSERVATIONS OF THE GM AURIGAE CIRCUMSTELLAR DISK
}

\author{
G. SCHNEIDER \\ Steward Observatory, University of Arizona, 933 North Cherry Avenue, Tucson, AZ 85721-0065; gschneider@as.arizona.edu \\ K. WoOD \\ School of Physics and Astronomy, University of St. Andrews, Fife, KY16 9AJ, Scotland, UK; kw25@st-andrews.ac.uk \\ M. D. Silverstone AND D. C. Hines \\ Steward Observatory, University of Arizona, 933 North Cherry Avenue, Tucson, AZ 85721-0065; \\ murray@as.arizona.edu,dhines@as.arizona.edu \\ D. W. KOERnER \\ Department of of Physics and Astronomy, Northern Arizona University, Box 6010, Flagstaff, AZ 86011; \\ David.Koerner@nau.edu \\ B. A. Whitney \\ Space Science Institute, 3100 Marine Street, Suite A353, Boulder, CO 80303-1058; bwhitney@colorado.edu \\ J. E. BJORKMAN \\ Ritter Observatory, Department of Physics and Astronomy, University of Toledo, Toledo, OH 43606; jon@astro.utoledo.edu \\ AND \\ P. J. LOWRANCE \\ Infrared Processing and Analysis Center, California Institute of Technology, 770 South Wilson Avenue, Pasadena, CA 91125; \\ lowrance@ipac.caltech.edu \\ Received 2002 October 2; accepted 2002 November 21
}

\begin{abstract}
We present Hubble Space Telescope Near Infrared Camera and Multi-Object Spectrometer (NICMOS) coronagraphic observations of the environment in the region of the young star-disk system GM Aurigae. Scattered near-infrared light in two spectral bands (F110W $=1.1 \mu \mathrm{m}$ and $\mathrm{F} 160 \mathrm{~W}=1.6 \mu \mathrm{m})$ trace the morphology of circumstellar dust to a distance of $\sim 700 \mathrm{AU}$ from the star. An $\sim 300$ AU radius outwardly flared disk inclined $50^{\circ}-55^{\circ}$ to the plane of the sky surrounded by a tenuous envelope is seen in the NICMOS images, confirming the size and suspected flared nature of the disk suggested by earlier $\mathrm{CO}$ and optical observations. The NICMOS images probe the disk region with spatial resolutions of $\sim 00^{\prime \prime} 1$ at radial distances of 0 ".35 to $\sim 4$ ".5-5".0 from the largely unobscured $\left(A_{v}<0.5\right)$ central star. The midplane of the disk 1".3 from GM Aurigae is revealed in silhouette against the previously unseen "lower" portion of the illuminated disk along its minor axis (as projected onto the sky). We comment on surface brightness profiles along the disk major and minor axes, as well as isophotal maps of the disk. From these photometric data we have measured the integrated flux density of the disk, beyond the instrumental inner radius of 0 !" 35 , as 8.0 and $9.3 \mathrm{mJy}$ $( \pm 20 \%)$ at $\mathrm{F} 110 \mathrm{~W}$ and $\mathrm{F} 160 \mathrm{~W}$, respectively, corresponding to disk scattering fractions of $L_{\text {disk }} / L *=0.025$ $( \pm 20 \%)$ in both bands. By fitting the photometric properties of the disk to a scattered-light model, we estimate the disk mass to be $\sim 0.04 M_{\odot}$. Additionally, we find two diffuse red polar lobes along the disk minor axis, likely the result of a bipolar outflow, at distances of \pm 3 ".8 from GM Aurigae ( $~ 900$ AU with our inferred inclination) with peak $H$-band surface brightnesses of $\sim 14 \mu \mathrm{Jy} \operatorname{arcsec}^{-2}$. We also note the existence of a broad $\left(\sim 3^{\prime \prime}\right.$ wide) band of blue material within the NICMOS field of view, spatially coincident with and extending at least $12^{\prime \prime}$ from the northeast outer region of the disk major axis.
\end{abstract}

Key words: circumstellar matter — stars: individual (GM Aurigae)

\section{INTRODUCTION}

Our understanding of the formation and evolutionary processes that may give rise to exoplanetary systems has advanced dramatically in the last few decades. Just as protostellar clouds are progenitors of stars and their protoplanetary debris systems, it is in those dusty circumstellar environments where nascent planetary systems may be coevolving. Since the detection of large amounts of dust around Vega-like stars by IRAS (Aumann 1985), as inferred from their thermal infrared excesses above the photospheric levels, many studies have concentrated on hotter, more massive stars. Such stars illuminate material at large physical distances, allowing detailed observations even with modest angular resolution. Lower mass stars (late $\mathrm{G}, \mathrm{K}$, and $\mathrm{M}$ types) far outnumber the higher mass A and F stars, have much longer lifetimes, and, despite their cooler photospheres, maintain reasonably large habitable zones within relatively benign radiation environments. They therefore provide an important sample for studying the number and nature of planetary systems, both from a fundamental understanding of formation mechanisms and as candidates for potentially life-bearing systems. The high-resolution, high-contrast imaging capabilities of the Hubble Space Telescope (HST) coronagraphic instruments are enabling us to examine these critically important objects and their environments in unprecedented detail (Schneider 2003).

GM Aurigae (R.A. $=04^{\mathrm{h}} 55^{\mathrm{m}} 10^{\mathrm{s}} .2$, decl. $=+30^{\circ} 31^{\prime} 58^{\prime \prime}$ [J2000.0], $d \sim 140 \mathrm{pc}$ ) is a classical T Tauri star (cTTS) of spectral type K7 V (White et al. 1999). Cohen \& Kuhi (1979) 
found GM Aurigae's effective temperature to be $3970 \mathrm{~K}$, and its age was estimated by Siess, Forestini, \& Bertout (1999) as $1.3 \pm 0.3$ Myr. Duplicity studies (e.g., Konig, Neuhauser, \& Stelzer (2001) indicate GM Aurigae is a single star, and its mass has been determined dynamically by Simon, Dutrey, \& Guilloteau (2000) as $0.84 \pm 0.05 M_{\odot}$. GM Aurigae exhibits strong $\mathrm{H} \alpha$ (White \& Ghez 2001), as well as Paschen- $\beta$ and Bracket- $\gamma$ emission, as is common for young stars (Folha \& Emerson 2001). Its near-IR apparent magnitudes as found from the Two Micron All Sky Survey (2MASS) were reported $^{1}$ as $J=9.315 \pm 0.036$ and $H=8.578 \pm 0.026$ though, as is typical for cTT stars, GM Aurigae exhibits intrinsic brightness variations of a few tenths of a magnitude in the near-IR (e.g., Bouvier et al. 1993, 1995).

The presence of a large amount of dust around GM Aurigae was established by Beckwith et al. (1990) in a $1.3 \mathrm{~mm}$ continuum survey of 86 pre-main-sequence (PMS) stars in the Taurus-Aurigae region. Koerner, Sargent, \& Beckwith (1993) reported ${ }^{13} \mathrm{CO}(2 \rightarrow 1)$ and $1.4 \mathrm{~mm}$ continuum emission, which they interpreted as originating from the core of a $950 \pm 530$ AU disk of gas and dust coincident with the position of GM Aurigae. From their CO observations they found differential rotation in the velocity field about an axis at $\mathrm{PA}=50^{\circ}$, consistent with Keplerian rotation. Dutrey et al. (1998) resolved the dust disk with 1.3 and $2.7 \mathrm{~mm}$ continuum emission by using the IRAM interferometer and confirmed the kinematic results of Koerner et al. (1993). They inferred a minimum radius for the disk of 200 AU (1!"4) and constrained the inclination of the rotation axis to $57^{\circ} \pm 5^{\circ}$ from the line of sight. The disk was detected but unresolved with a total flux density of $22 \mathrm{mJy}$ in a $2.7 \mathrm{~mm}$ BIMA survey conducted by Looney \& Mundy (2000), though they suggested a slight extension perpendicular to the large-scale CO structure.

Polarized $850 \mu \mathrm{m}$ emission (at the 3\% level) was reported by Tamura et al. (1999) and interpreted as arising from thermal reradiation by magnetically aligned dust grains in the disk. Skrutskie et al. (1990) found significant mid-IR emission over the stellar photospheric level, indicative of a reradiating circumstellar dust disk. The relatively flat midIR excess and millimeter emission (Koerner et al. 1993) were fitted to a model spectral energy distribution (SED) by Chiang \& Goldreich (1997) assuming a passive face-on disk. More recent work by Chiang \& Goldreich (1999) suggested a polar inclination of $\sim 60^{\circ}$ to the line of sight and a visual extinction of GM Aurigae by its circumstellar disk of 0.5 mag, larger than the $A_{v}$ of 0.14 suggested by Cohen \& Kuhi (1979) or more recently $A_{v}=0.31$ by Gullbring et al. (1998). Therein Chiang \& Goldreich (1999) described GM Aurigae as a "class II source, only slightly obscured by its circumstellar disk" which, given its inferred angular extent of a few arcseconds, has made its direct imaging in scattered light observationally challenging.

Stapelfeldt et al. (1997) reported imaging the GM Aurigae disk in the visible with the HST Wide Field and Planetary Camera 2 (WFPC2). Their imaging observations demonstrated the existence of an upwardly flared disk with a morphology implicating an inclination to the line of sight of $\sim 65^{\circ}$, similar to the inclination deduced by Dutrey et al.

\footnotetext{
${ }^{1}$ See http://www.ipac.caltech.edu/2mass/releases/second/doc/ explsup.html.
}

(1998). They estimated a large disk-scattering fraction with $\sim 8 \%$ of the visible light from the GM Aurigae system provided by the circumstellar material.

Koerner et al. (1998) confirmed both the flared nature of the disk and the suggested inclination with the HST Near Infrared Camera and Multi-Object Spectrometer (NICMOS), utilizing its coronagraphic imaging mode at 1.1 and $1.6 \mu \mathrm{m}$ in a preliminary analysis of those data before the coronagraphic imaging mode of NICMOS was fully characterized and calibrated. Here, with those instrumental characteristics now well understood, we discuss in greater detail these spatially resolved NICMOS imaging observations, which elucidate the morphology and geometrical characteristics of the scattered light disk. We present the first imaging of the lower portion of the disk, partially shadowed by optically thick material in the midplane. With these newly revealed features, the GM Aurigae disk appears to have an intrinsic morphology similar to HH 30 (Cotera et al. 2001), Haro 6-5B (Padgett et al. 1999), and the companion to HK Tau (Stapelfeldt et al. 1998). With these images we also present evidence for the existence of a bipolar outflow at an early stage of collimation. Such outflows, though undetected in the less sensitive optical GM Aurigae observations of Stapelfeldt et al. (1997), have been observed in the circumstellar environments of similar stars (such as HH 30), as well as stars of higher mass and somewhat older ages (e.g., the Herbig Ae/Be stars SU Aurigae; Grady et al. 2001) and HD 163296 (Grady et al. 2000).

\section{OBSERVATIONS}

Observations of the GM Aurigae circumstellar disk were obtained with the HST NICMOS coronagraph on UT 1998 March 25. Two spectral filters were employed: F110W (central $\quad$ wavelength $=1.0998 \mu \mathrm{m}, \quad \mathrm{FWHM}=0.5915 \mu \mathrm{m}$; roughly similar to $J$ band) and F160W (central wavelength $=1.5940 \mu \mathrm{m}, \mathrm{FWHM}=0.4000 \mu \mathrm{m}$; a close analog to $H$ band). The spacecraft was rolled to a single NICMOS camera 2 field orientation angle (position angle of the image $+Y$-axis $=214.51$ east of north) for all images. With each filter two STEP128 NSAMP $=14$ MULTIACCUM imaging sequences (Schultz et al. 2001) were executed, yielding total integration times of $1279.86 \mathrm{~s}$ after combining the reduced images in each passband.

The NICMOS coronagraphic optics are in camera 2, which provides a $256 \times 256$ pixel field of view at a scale of $\sim 76$ mas pixel ${ }^{-1}$. A star of F110W and F160W magnitude 20.4 and 19.4 appeared in the field of view, 12!"7 to the northeast of GM Aurigae, from which the FWHM of the coronagraphic point-spread functions (PSFs) were contemporaneously determined as 0.1098 and 0 "! 132 , respectively. ${ }^{2}$

\footnotetext{
${ }^{2}$ NICMOS coronagraphy is performed with the reimaged focal plane at the detector moved to a marginally different position than used for direct imaging to maximize coronagraphic contrast. In addition, the HST focus interface to the science instruments, including NICMOS, changes by very small amounts with the spacecraft orbit and attitude, which affects the fine structure of the PSF (Schneider et al. 2001). The instantaneous spatial scale of a resolution element may be determined (for a given spectral band) from unocculted target acquisition images, closely spaced in time preceding the coronagraphic images. Here, we have determined this contemporaneously for each passband (expressed as the FWHM of the PSF from an unresolved point source) by using the well-exposed star that appeared serendipitously in the field of view.
} 
These images were acquired as part of the NICMOS IDT Environments of Nearby Stars (EONS) program (GTO/ 7233; B. A. Smith, principal investigator) and were the first NICMOS coronagraphic science images obtained following the installation of the instrument in HST. The target was placed into the 0".3 ( 4 pixel) radius coronagraphic " hole" by an autonomous on-board acquisition process. A posteriori, GM Aurigae was found to have been decentered by ( $\Delta X=0.49, \Delta Y=-0.01)$ pixels from the low scatter point of the coronagraphic system, the offset resulting from a bug in the target acquisition flight software that was fixed soon after this observation.

\section{DATA CALIBRATION, REDUCTION, AND PROCESSING}

\subsection{Photometric Calibration}

Count rate images corrected for the wavelengthdependent instrumental flat-field response, pixeldependent dark currents, and detector nonlinearities were created from the raw MULTIACCUM frames by using an IDL-based analog ${ }^{3}$ to the IRAF STSDAS NICMOS CALNICA image reduction software (Stobie et al. 1998), and calibration reference files, both created by the NICMOS IDT. Known underresponsive pixels and those with excessive dark currents were replaced using twodimensional Gaussian-weighted interpolation of neighboring pixels. In doing so we used weighting radii of 3 and 5 pixels (appropriate for the F110W and F160W resolution elements, respectively, as sampled with the camera 2 pixels). The two count rate images for each filter were averaged and image artifacts not compensated for in the CALNICA processing ${ }^{4}$ were quantitatively characterized and removed. These electronically induced artifacts include zero-level offsets between the four detector quadrants, elevated count rates along a strip orthogonal to the readout direction encompassing deeply exposed pixels, low-amplitude electronic "echoes" of deeply exposed targets at the same locations in the other detector quadrants, and residual imprints of the detector flat field from uncompensated pedestal effects.

The corrected count rate images were converted to photometric units based upon the absolute on-orbit photometric calibration determined by the NICMOS IDT of $2.031 \mu \mathrm{Jy}^{-1}$ $\mathrm{ADU}^{-1} \mathrm{~s}^{-1}$ pixel $^{-1}$ for F110W and $2.190 \mu \mathrm{Jy}^{-1} \mathrm{ADU}^{-1} \mathrm{~s}^{-1}$ pixel $^{-1}$ for F160W (M. Rieke 2002, private communication). The photometric zero points to convert to in-band magnitudes, ascertained from the same calibrations, yield $1775 \mathrm{Jy}(\mathrm{F} 110 \mathrm{~W})$ and $1083 \mathrm{Jy}(\mathrm{F} 160 \mathrm{~W})$ for the total energy for a zero-magnitude (Vega system) star.

\subsection{Reference Point-Spread Functions}

The NICMOS coronagraph provides gains in image contrast of factors of a few to about 10 within a few arcseconds of an occulted star by reducing the intensity of the diffracted energy in the wings of the stellar point-spread function and the "downstream" instrumental scatter from the masked PSF core (Schneider et al. 1998). Further improvements, of

\footnotetext{
${ }^{3}$ IDL is a registered trademark of Research Systems Incorporated, a Kodak company.

${ }^{4}$ See http://www.stsci.edu/hst/nicmos/performance/anomalies.
}

up to factors of $\sim 50$, are obtained by subtracting wellmatched, high S/N (signal-to-noise ratio), template PSFs of reference stars. Ideally, a reference coronagraphic PSF to be subtracted from a target image should be observed at the same subpixel location within the coronagraphic hole. Normally, this is achieved to a very high degree of precision by the on-board autonomous target acquisition process. However, this process was flawed at the time of the GM Aurigae observation.

During the HST NICMOS SMOV-2 calibration program we obtained deeply exposed reference PSFs in the F160W filter rastered in a subpixel grid in the coronagraph (SMOV/7052; G. Schneider, principal investigator). Fortuitously, in that grid, three MULTIACCUM images of a bright reference star (BD $+032964, H=5.0)$ were positioned at $(\Delta X=+0.50, \Delta Y=0.00)$ pixels with respect to the low scatter point of the coronagraphic system. To the achievable pointing precision, this is differentially identical to the mispointed GM Aurigae observation. Hence, for our F160W observations we created a reference PSF from these observations of BD +032964 for subsequent subtraction from the GM Aurigae data set. Unfortunately, an equivalent raster was not carried out under the F110W filter. The sensitivity to decentering, however, is far less severe at $1.1 \mu \mathrm{m}$ than at $1.6 \mu \mathrm{m}$ primarily because of the smaller spatial scale $(68 \%)$ of the shorter wavelength PSF (Schneider et al. 2001). For our F110W observation we created a holecentered reference PSF obtained in the same calibration program (from 10 raw images) with the recognition that the residual pointing misalignment would be the principal source of systematic error limiting the background subtraction at small radii near GM Aurigae. Reference PSF images were reduced and calibrated from this data set in the same manner as were our target images.

\subsection{Geometrical Correction, Rectification, and Scale}

The NICMOS camera $2 X / Y$ image plane pixel scales differ by $\sim 0.9 \%$. Additionally, the absolute scales varied by small amounts during the early part of the HST Cycle 7 mission. ${ }^{5}$ As a result the image scales for the BD +032964 reference PSFs and the target (GM Aurigae) images differ and required geometrical rectification and rescaling before the reference PSFs could be subtracted. For the epochs of these observations the NICMOS camera 2 pixel scales were for 1997 July 22 (BD +032964) (76.11, 75.43) mas pixel ${ }^{-1}$ and for 1998 March 25 (GM Aurigae) $(75.99,75.31)$ mas pixel ${ }^{-1}$.

The F110W and F160W photometrically calibrated images were resampled on a common grid to square up the pixels on the sky (adjusting the $Y$-scale to match the $X$-scale) and the target images were resampled to match their reference PSF image scale. Image resampling was done by sinc function apodized bicubic interpolation with the NICMOS IDT IDP3 software (Schneider \& Stobie 2002), so all images were geometrically rectified to have $76.11 \times 76.11$ mas $^{2}$ pixels, with the total (area integrated) flux conserved in the resampling process.

\footnotetext{
${ }^{5}$ See http://www.stsci.edu/hst/nicmos/performance/platescale/ rel_platescale.html.
} 


\subsection{Target/PSF Image Registration, Scaling, and Subtraction}

After correcting for the camera 2 geometrical distortions, the F110W and F160W reference PSF images were registered, flux-scaled, and subtracted from the corresponding target images (also using IDP3). PSF subtraction residuals in the difference images are spatially correlated over multiple pixel scales in the radial direction along (and immediately adjacent to) the bright diffraction artifacts from the $H S T$ secondary mirror supports (Schneider \& Silverstone 2002). The reference PSF subtractions were constrained to minimize the energy in the diffraction spike residuals by simultaneously adjusting the reference PSF star brightness and position. Because of small anisotropic secular instabilities in the HST plus NICMOS instrumental system, a globally optimized solution over the entire field cannot be obtained and is the dominant source of uncertainty in ascertaining the correct flux density scaling for the reference PSF (Schneider et al. 2001). Here we found the ratio of the GM Aurigae photospheric flux density to that of BD +032964 was $0.0380 \pm 0.0008$ at $\mathrm{F} 110 \mathrm{~W}$ and $0.0325 \pm 0.0006$ at F160W. BD +032964, with spectral type G2 V, has a spectral energy distribution under the F110W and F160W filter passbands that is very similar to GM Aurigae. Hence, colordependent differential PSF structures (arising from the small mismatch in spectral types) are very small and do not significantly contribute to the imperfections in nulling the underlying stellar flux (e.g., Weinberger et al. 1999, 2002).

Following Schneider et al. (2001) we estimated the uncertainty in the flux density of the disk arising from the uncertainty in the flux scaling of the target and reference stars and in the presence of imperfectly nulled artifacts from the diffraction spikes. ${ }^{6}$ We find this uncertainty is $\lesssim 20 \%$ of the total flux density integrated outward from any inner radius beyond $0 . " 4$ in both spectral bands.

\section{GM AURIGAE SCATTERED LIGHT DISK}

The morphology of the light scattered in the circumstellar region of GM Aurigae displays the characteristic pattern of a flared disk (Whitney \& Hartmann 1992) and is shown in a series of four images presented in increasing levels of sensitivity in Figure $1 a-1 d$. GM Aurigae itself is occulted in the 0 ". 3 radius coronagraphic hole (black circle). The upper portion of an outwardly flared disk, inclined to the line of sight, is readily seen in both the F110W and F160W spectral bands (Fig. 1a). The disk is bilaterally symmetric, brighter "forward" (to the north) of the major axis, and mirrored in its surface brightness distribution about the minor axis. The forward scattering edge of the lower portion of the disk, "below" the higher opacity midplane in the region of the minor axis to the north, becomes visible at lower flux densities (Fig. 1b, where the central portion of the disk is saturated). At lower surface brightness (Fig. 1c-1d) a kinked broad "ribbon" of blue material extends to the northeast

${ }^{6}$ Unlike other stars in our disk survey, the GM Aurigae images were acquired with only a single orientation of the NICMOS camera 2 coronagraphic field. As a result, the regions of the disk coincident with the diffraction spike residuals, after PSF subtraction, cannot be replaced by data obtained at a second field orientation, which would have sampled the affected regions of the disk.

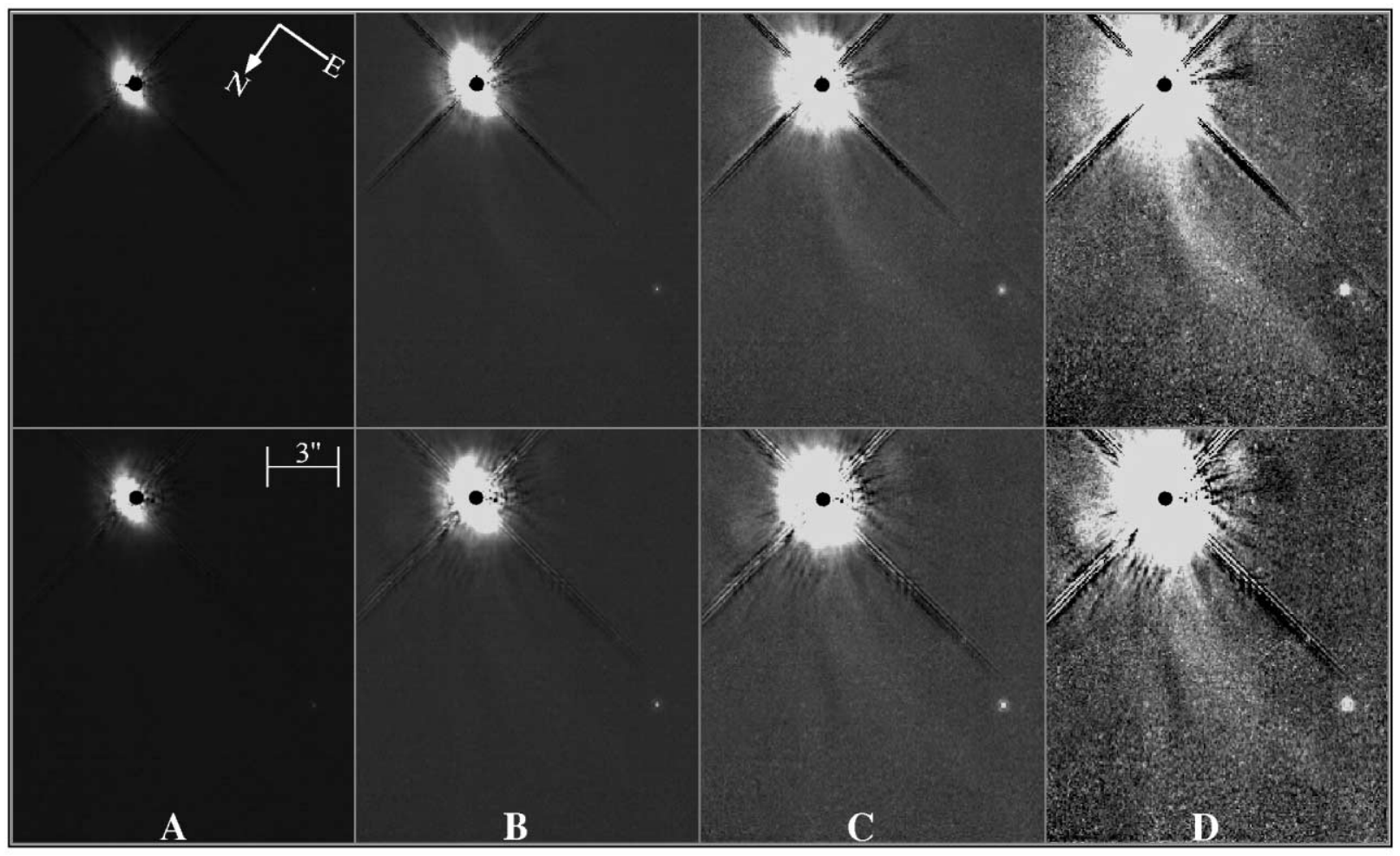

FIG. 1.-NICMOS Camera 2 PSF-subtracted coronagraphic images of the GM Aurigae scattered light disk. To accommodate the very large dynamic range

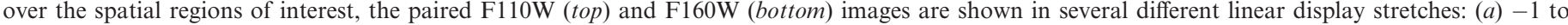
$+10 \mu \mathrm{Jy}$ per pixel; $(b)-0.5$ to $+2 \mu \mathrm{Jy}$ per pixel; $(c)-0.2$ to $+0.5 \mu \mathrm{Jy}$ per pixel; $(d)-0.05$ to $+0.1 \mu \mathrm{Jy}$ per pixel. 
for a distance of at least $12^{\prime \prime}$, declining in brightness in both F110W and F160W with increasing distance from GM Aurigae. Finally, diffuse red polar lobes (likely of outflow material) are seen symmetrically along the minor axis at distances of $\sim 3$ ".5- $4^{\prime \prime}$, with peak $H$-band surface brightnessess $\sim 0.14 \mu \mathrm{Jy}$ per camera 2 pixel. Figure 2 , a two-color combination of the F110W and F160W images, shows these features and their "colors," in two logarithmic stretches.

The morphology of the circumstellar light from the inner portions of the disk (to $\sim 1^{\prime \prime}$ along the minor axis in the forward-scattering direction and $\sim 1 "$ "6 along the major axis) traced by earlier highest spatial resolution (F555W) WFPC2 observations of Stapelfeldt et al. (1997) is in general agreement with the NICMOS images. Material in the circumstellar environment beyond these distances from GM Auriage remains undetected in the less sensitive WFPC2 observa- tions. Those noncoronagraphic observations and those in the other WFPC2 bands (F675W and F814W) are of significantly lower signal-to-noise ratio, have more limited dynamic range (due to the sampling mode of the instrument), and are adversely affected by larger relative amplitude PSF-subtraction residuals than the NICMOS data. For these reasons we confine our discussions of the disk features and base our scattered light models only on the NICMOS observations, which are of higher photometric fidelity. We note the disparity by a factor of 2 in the diskscattering fraction of $\sim 8 \%$ reported by Stapelfeldt et al. (1997) relative to our NICMOS measure of $\sim 4 \%$ (see $\S 4.1$ ). Rather than a strong intrinsic color difference under the two spatially adjacent passbands of WFPC2 (F814W) and NICMOS (F110W) filters, we suggest this is likely caused by systematic instrumental effects in the WFPC2 data.

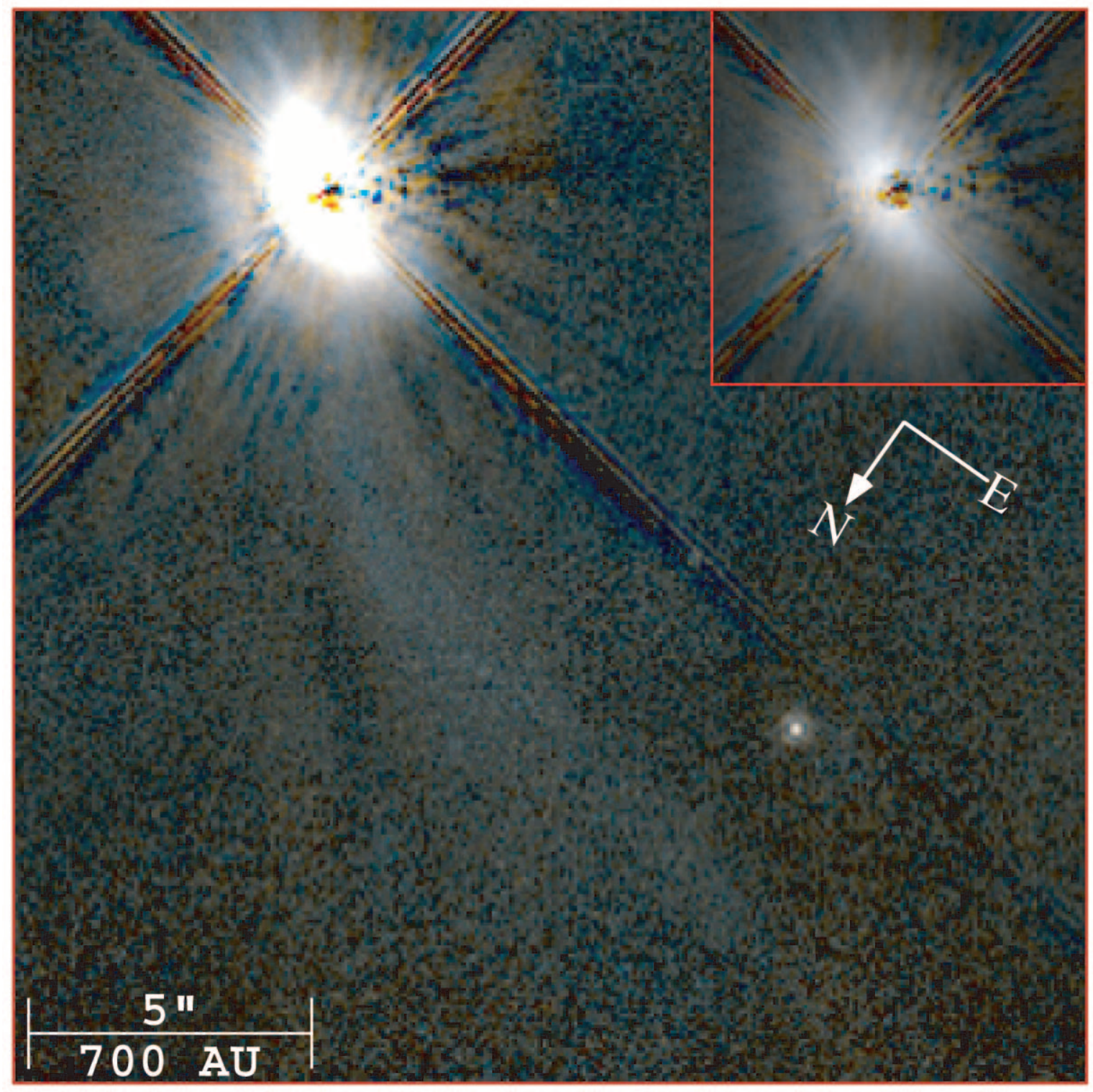

Fig. 2.-Wavelength-dependent morphology of the GM Aurigae circumstellar environment, illustrated in a two-color composite image derived from F110W (blue) and F160W (red) PSF-subtracted frames. The logarithmic display stretch of the full 19".4 × 19".3 field shows the polar lobes (red) along the disk minor axis, the ribbon of material extending $\sim 13^{\prime \prime}$ to the northeast (blue), and the field star used (in each band) to measure the FWHM of the coronagraphic focus PSF. The midplane of the disk and the upper edge of the lower (forward) scattering surface are readily seen at a lower sensitivity logarithmic stretch (inset). 


\subsection{Photometry of the Disk}

The photometric efficacy of the GM Aurigae minus BD +032964 PSF subtractions permits measurement of the flux density of the circumstellar disk to radial distances closely approaching the geometrical edge of the coronagraphic hole. We estimate the total flux density of the disk from 0 ". 35 to $5 . " 0$ to be $8.0 \mathrm{mJy}$ at F110W and $9.3 \mathrm{mJy}$ at F160W, both with uncertainties $\lesssim 20 \%$. We obtained two short (1.22 s) but high $\mathrm{S} / \mathrm{N}$ (each 320) F160W noncoronagraphic target acquisition images of GM Aurigae immediately prior to our coronagraphic exposures. After calibration we measured the F160W flux density of GM Aurigae as $0.344 \pm 0.007 \mathrm{Jy}$, or $H=8.74 \pm 0.02$, which is in agreement with the $H=8.73 \pm 0.16$ mag in Kenyon \& Hartmann (1995). We do not have a contemporaneous measure of the brightness of the star in the NICMOS F110W bands but adopt the $J$ magnitude of 9.37 from Kenyon \& Hartmann (1995) as a very close surrogate given the reproducibility of the F160W ( $H$ band) measure at the time of our observations. From this we find the disk-scattering fraction (ratio of light scattered by the disk to starlight), $L_{\text {disk }} / L_{*}$, is 0.025 $( \pm 20 \%)$ in both spectral bands. Thus, in the near-IR, $L_{\text {disk }} /$ $L_{*}$ is very similar to that found for the optically thick faceon disk around the K7 Ve cTTS TW Hydrae by Weinberger et al. (2002; with $L_{\text {disk }} / L_{*}=0.024$ and 0.021 for F110W and F160W, respectively). To facilitate flux density measurements of the GM Aurigae disk with other instrumental systems that might not probe as effectively to small radii, in Figure 3 we provide the integrated flux densities in both bands as a function of the inner radius sampled.

The southwest side of the disk beyond 4".5 from GM Aurigae along the major axis fell outside the NICMOS field of view. At that distance the disk major axis surface brightness declines to $23 \mu \mathrm{Jy} \operatorname{arcsec}^{-2}$ in F110W and to $17 \mu \mathrm{Jy}$ $\operatorname{arcsec}^{-2}$ in F160W. The disk isophotes, shown in contour maps for both the F110W and F160W images in Figure $4 a-$

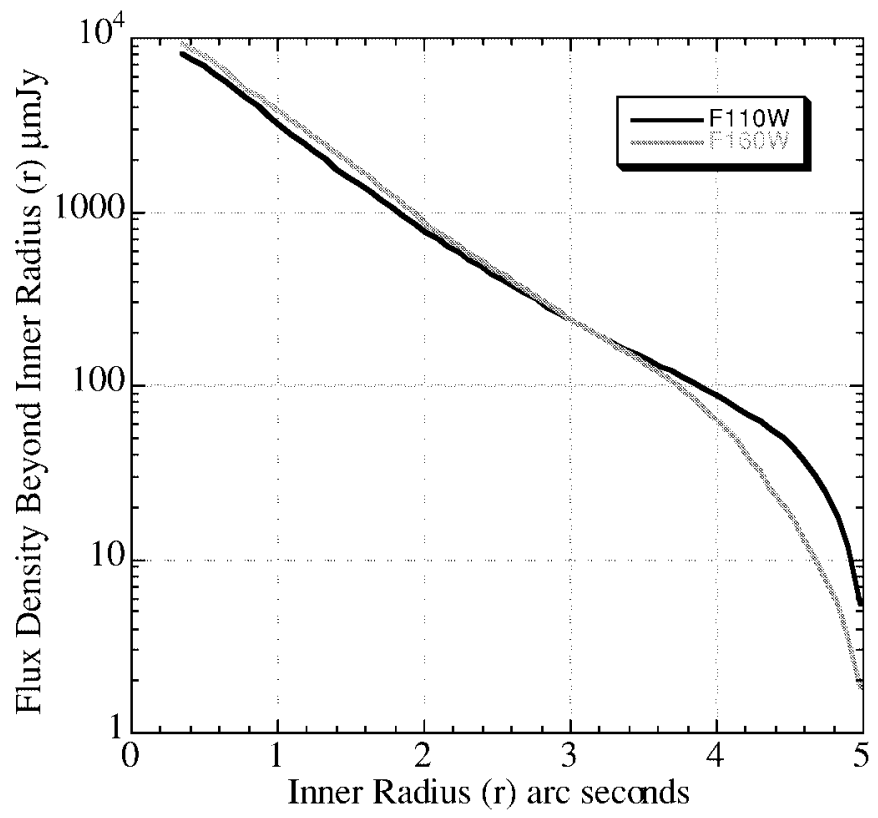

FIG. 3.-Area-integrated F110W and F160W flux densities of the GM Aurigae circumstellar disk measured radially outward from the inner radii indicated to a distance of $5^{\prime \prime}$. Photometric uncertainties are $\lesssim 20 \%$ of the measured flux densities.
$4 b$, respectively, exhibit a high degree of bilateral symmetry, and the northeast side of the disk extends to approximately $5^{\prime \prime}$. The outer edge of the detectable disk adjoining the northeast major axis is spatially coincident with a broad and diffuse "ribbon" of material, which makes the determination of the full extent of the disk difficult, but it would likely be $\sim 5^{\prime \prime}$ in the absence of this material.

The position angle of the disk minor axis in the direction of forward scattering was found to be $328^{\circ} .5 \pm 2.5$ (see $\S 5.1$ ). The radial surface brightness profile of the disk, measured along a sector $45^{\circ}$ in azimuthal extent centered on the minor axis (toward the northwest), is shown in Figure 5 for both filters. These brightness profiles are globally well fitted to power laws with brightness falloffs of $r^{-2.9}$ and $r^{-3.5}$ for F110W and F160W, respectively. Some statistically significant deviations from these simple power laws are apparent and are understood through an examination of the images themselves.

The surface brightness of the northwest minor axis sector of the disk is depressed from the power-law fit in a radial zone $\sim 1$ !.3 from GM Aurigae, most notably in the F160W filter. This decrement in surface brightness is likely due to higher opacity material in the midplane of the disk that, because of the viewing geometry, partially obscures the lower portion of the disk. The surface brightness in F160W then rises significantly above the $r^{-3.5}$ power-law fit with a maximum deviation at $\sim 1$ "' 85 . The F160W flux densities in this region of $\sim 150 \mu \mathrm{Jy} \operatorname{arcsec}^{-2}$ likely arise from scattering off the upper surface of the lower portion of the disk unobscured by material in the midplane.

\subsection{Axial Flux Densities}

In Figure 6 we show the F110W and F160W flux densities along both the disk minor and major axes, in both directions outward from GM Aurigae, as functions of distance from the star. The flux densities shown are measured in 1 pixelwide radial increments in regions $1^{\prime \prime}$ in extent centered on and orthogonal to the axes. In the direction of forward scattering (along the minor axis), the flux density decrement at about -1 ".3, likely due to shadowing by the midplane, and the relative enhancement from the forward-scattering surface of the lower part of the disk at about -1 ".85 are readily apparent. The flux density in the "positive" direction along the minor axis (in Fig. 6), where the line of sight is to the "back" of the upper portion of the disk, falls off more rapidly. In both directions localized brightness enhancements are seen in F160W at about \pm 3 ".5-4", which we interpret as weakly collimated material from a bipolar outflow (see $\S 7$ ). As one might expect, none of these features are seen along the disk major axis (though the major axis toward the southwest is truncated by the edge of the field of view). Unlike the overall "front-to-back" brightness asymmetry along the minor axis, we find the radial brightness along the major axis reflectively symmetric about GM Aurigae.

\section{SCATTERED LIGHT MODEL}

We modeled the NICMOS scattered light images with passively heated disks to determine the geometry (orientation, inclination, and size) and physical characteristics (disk mass, scale height, and envelope infall rate) of the GM Aurigae circumstellar disk. The properties of the disk grains govern the wavelength-dependent width of the dust lane, the spatial variation of the scattered light, and the slope of the 


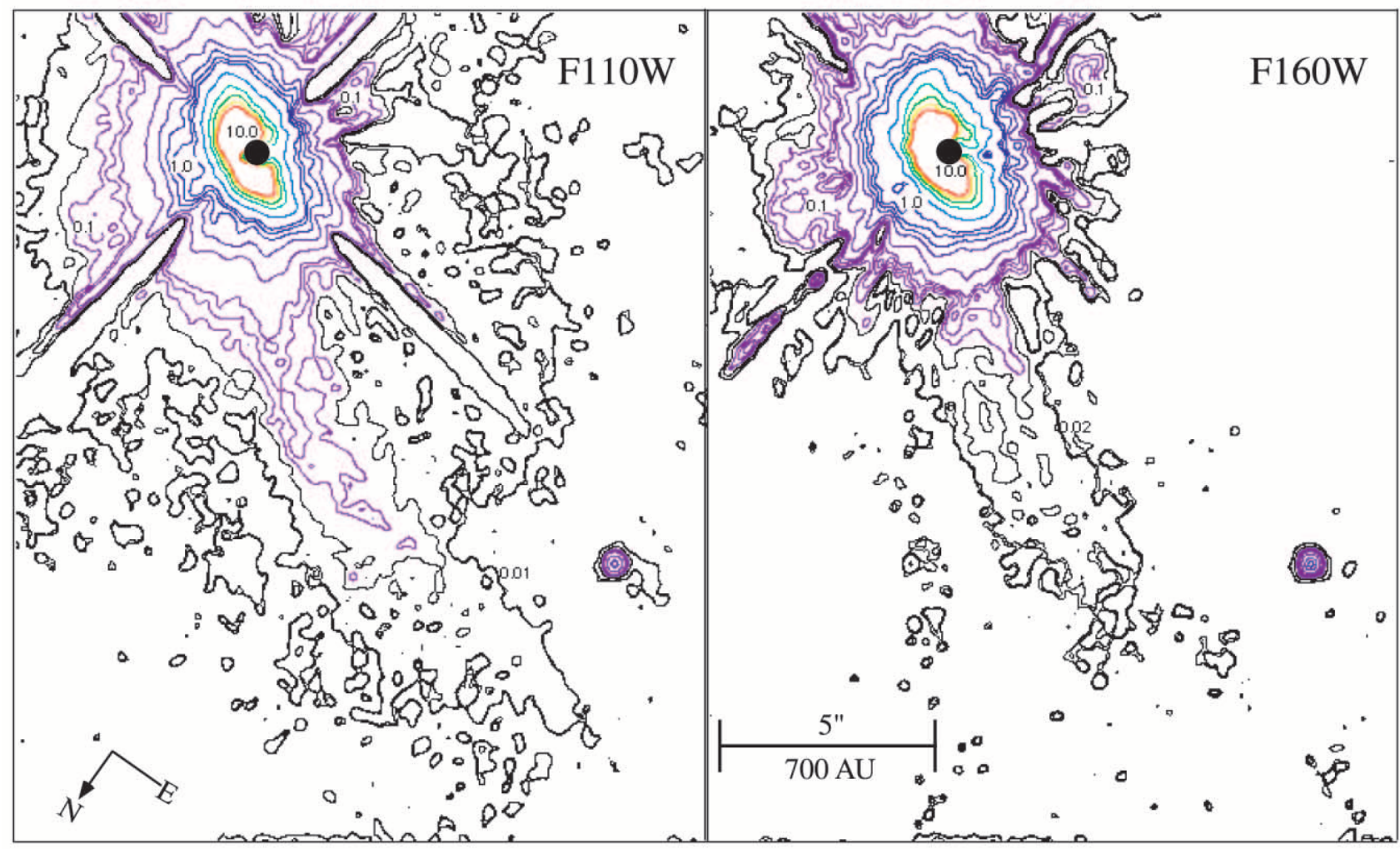

FIG. 4.- Isophotal contour plots of the GM Aurigae disk, after applying a $3 \times 3$ pixel boxcar-smoothing kernel, illustrating the flux density levels of the features discussed in the text. Contour levels and intervals in microjanksys per pixel are for F110W (left): 0.0085, 0.01-0.1 at 0.02, 0.2-1.0 at 0.2, 2-10 at 2, and for F160W (right): $0.017,0.02-0.12$ at $0.02,0.2-1.0$ at 0.2 , and $2-10$ at 2 .

long-wavelength continuum of the spectral energy distribution. The disk dust opacity exhibits a shallower wavelength dependence than interstellar material grains (e.g., Cardelli, Clayton, \& Mathis 1988), and this is determined from the slope of the long-wavelength continuum SED (e.g., Beck-

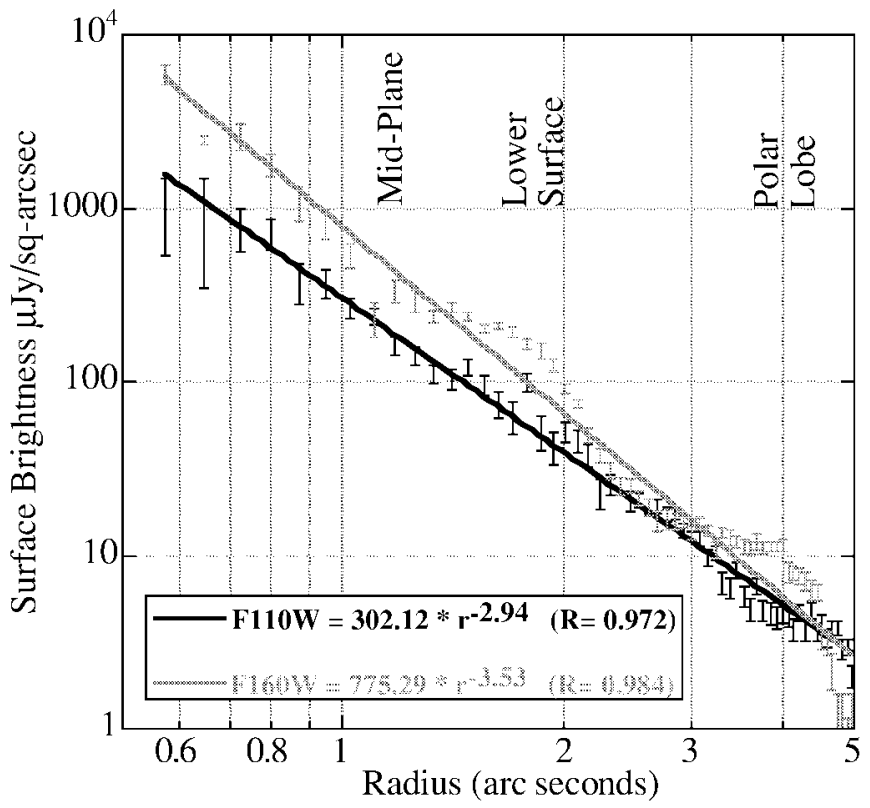

FIG. 5.-Median F110W and F160W radial surface brightness profiles of a $45^{\circ}$ azimuthal sector of the disk centered on the minor axis to the northwest. Error bars represent the internal standard error of the flux density measured about the median at each measured radius. with et al. 1990; Beckwith \& Sargent 1991). As with many other classical T Tauri stars, the slope of GM Aurigae's submillimeter continuum suggests a dust opacity $\kappa \sim \lambda^{-1}$, indicating dust grains in the disk are larger than those in the interstellar medium. Recent observations indicate that with similar disks the width of the dust lane decreases with increasing wavelength. Scattered light and SED models for HH 30 IRS (Cotera et al. 2001; Wood et al. 2001) demonstrate that optical through near-IR images of edge-on disks can be used to determine the wavelength dependence of the dust opacity within the disk. The surface brightness distribution of the light scattered is related to the dust-scattering phase function.

To model both the light scattered by the disk and its spectral energy distribution ( $(6)$ we used the dust model described in Wood et al. (2002), which has a shallower wavelength-dependent opacity than the canonical interstellar medium extinction curve (e.g., Cardelli, Clayton, \& Mathis (1989). To fit both the NICMOS scattered light images and the SED we varied the viewing geometry for the adopted dust model (but see Burrows et al. 1996 for scattered light models that investigate changing grain parameters in the case of the HH 30 IRS disk). The grain parameters we used are from the grain model described in Wood et al. (2002), which were used successfully to reproduce the HH 30 IRS images and its SED. The phase function asymmetry parameter for this model is close to that obtained by Burrows et al. (1996).

We adopted a flared disk structure (e.g. Shakura \& Sunyaev 1973) with a radius of $300 \mathrm{AU}$ and a low-density outer envelope of radius $1000 \mathrm{AU}$ such as was required to fit the HH 30 nearly edge-on disk (Wood et al. 1998; Cotera et 


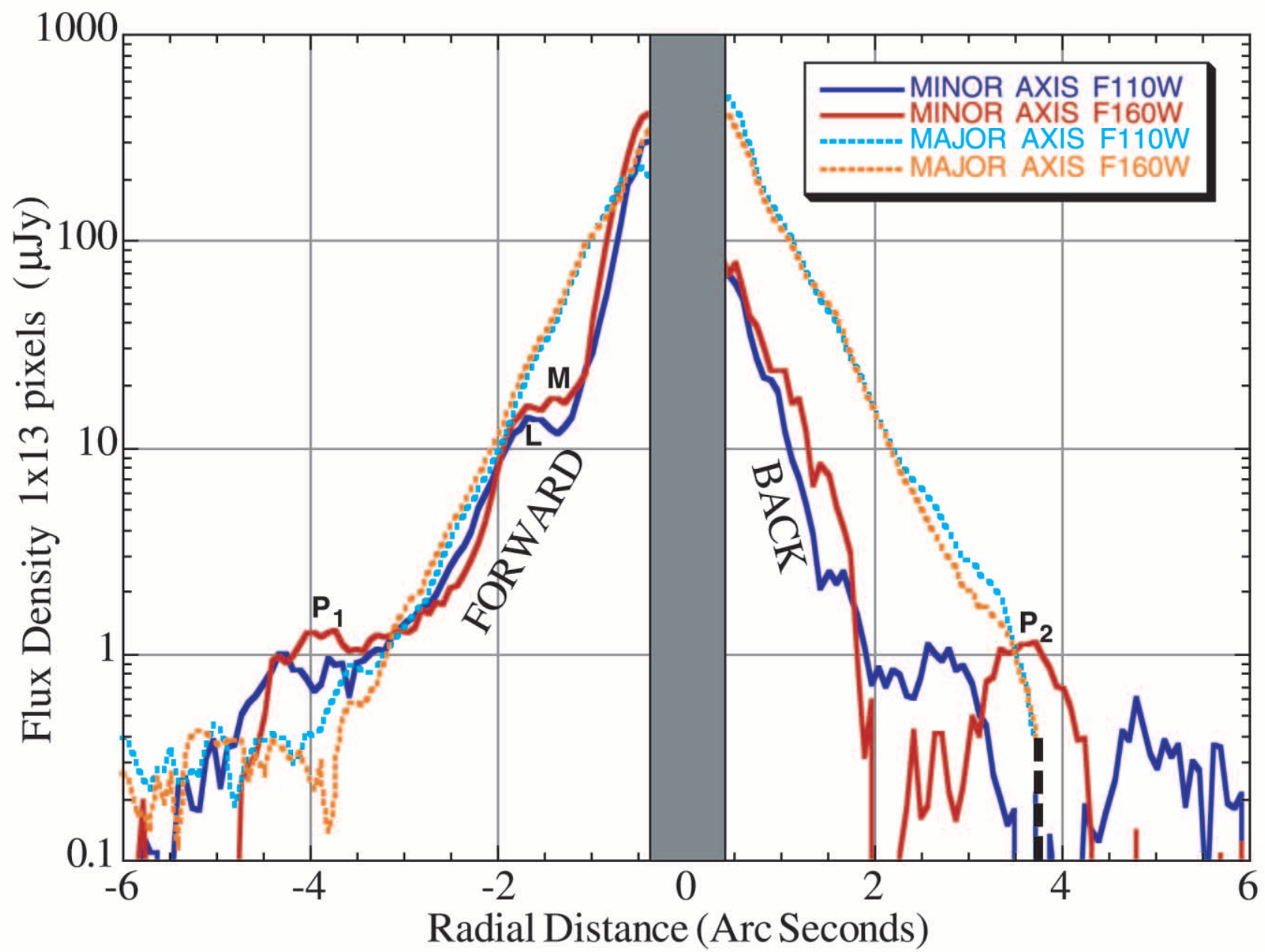

FIG. 6.-F110W and F160W flux densities along the GM Aurigae disk major and minor axes. Flux densities were measured in $1 \times 13$ pixel $\left(0{ }^{\prime \prime} 076 \times 1\right.$ (") regions, with the long axis of the radially incrementing photometric aperture orthogonal to the disk axes. M, at -1 "! 3 , denotes the region of the disk shadowed by the midplane. L, at -1 "' 85 , indicates the visible scattering surface of the lower portion of the disk. The polar lobes, P1 and P2, with statistically identical F160W flux densities, are located symmetrically along the minor axis at \pm 3 ".85 from GM Aurigae. A small portion of the outer edge of the disk toward the southwest (positive radial direction) is truncated by the NICMOS field of view (black dashed line). The region in gray is obscured by the NICMOS coronagraphic optics.

al. 2001). For the disk

$$
\rho_{d}=\rho_{0}\left(R_{*} / \varpi\right)^{\alpha} \exp -\frac{1}{2}[z / h(\varpi)]^{2},
$$

where $\rho_{0}$ is the disk density extrapolated to the stellar surface, $\varpi$ is the radial coordinate in the disk midplane, and the scale height increases with radius, $h=h_{0}(\varpi / R *)^{\beta}$. We adopted $\beta=1.25$ and $\alpha=2.25$, giving a surface density $\Sigma \sim 1 / \varpi$, as derived in the hydrostatic disk structure models of D'Alessio et al. (1999).

For the outer envelope we used the rotational collapse geometry of Terebey, Shu, \& Cassen (1984). We also included a curved bipolar evacuated cavity in the envelope (Whitney \& Hartmann 1993). The cavity walls are curved, with their height scaling as $z_{\text {cav }} \sim \varpi^{2}$ and a half-opening angle of $20^{\circ}$ at the outer radius of the envelope. The very small mass in the remnant envelope was required to match the scattered light images. The scattered light models used the Monte Carlo techniques described by Whitney \& Hartmann (1992).

\subsection{Results from Fitting the Scattered Light Model}

The observed photometric properties of the GM Aurigae disk were closely reproduced using the scattered light model described in $\S 5$. Earlier attempts at using a "simplified" model (i.e., disk-only models without an extended envelope and infall cavity) failed to replicate the scattered light intensity pattern seen in the NICMOS images over the full radial range of sensitivity made available in those data, particularly at large distances from the star. Adopting the model described in $\S 5$, we treated as free parameters (1) the disk mass, (2) the inclination of the disk to the plane of the sky, (3) the scale height of the disk, and (4) the infall rate of the envelope. We then compared the morphology and flux densities produced for each model with the NICMOS images in both spectral bands. The set of model parameters that most closely replicated the observations required a disk of 0.04 $M_{\odot}$ inclined $50^{\circ}-55^{\circ}$ with respect to the plane of the sky (in very good agreement with Dutrey et al. 1998, wherein they found the inclination to be $57^{\circ} \pm 5^{\circ}$ ). The scale height parameter was found to be $h_{0}=0.008 R *$ (corresponding to $8 \mathrm{AU}$ at a distance of $100 \mathrm{AU}$ from the star, a factor of 2 smaller than that found for the HH 30 disk by Burrows et al. 1996 and Cotera et al. 2001). The tenuous outer envelope was characterized with an infall rate of $1.5 \times 10^{-7} M_{\odot} \mathrm{yr}^{-1}$, and the centrifugal radius was set to be the disk radius, $300 \mathrm{AU}$. This gives a total envelope mass of $0.001 M_{\odot}$. 
Additionally, as part of the fitting process, we found the position angle of the disk minor axis in the direction of forward scattering to be $\mathrm{PA}=328^{\circ} .5 \pm 2.5$.

In Figure 7 we show the best-fit models to the GM Aurigae disk alongside the observations. We present model and difference (observed minus model) images for the best-fit set of disk parameters with inclinations of both $50^{\circ}$ and $55^{\circ}$. The models closely reproduce the flux densities seen over most regions of the disk as illustrated in Figure 8, with cuts through the major and minor axes, comparing the $50^{\circ}$ inclination models with the observations in both NICMOS bands. The disk residuals after model subtraction are of very small amplitude and symmetrically close to our observational inner radius of 0 ". 35 for a disk inclination of $50^{\circ}$ in $H$ band. For the same inclination the model underpredicts the F110W flux density close to the star in the direction of the disk major axis. By increasing the disk inclination to $55^{\circ}$ we obtain a better match to the distribution of F110W light near the star along the major axis, but the model then underpredicts the amount of scattering in the forward direction toward the region containing the midplane. The model also marginally overestimates the flux density of light scattered from the portion of the disk "above" the star (i.e., toward the "back" of the disk along the minor axis) at distances of 0 ".6-1".4. The flux densities in the region of the midplane and of the visible scattering surface of the lower portion of the disk are correctly predicted by the model. However, this lower portion of the disk is wider (e.g., along the minor axis) than the model suggests, and the brightness distribution in the radial direction is not as sharply peaked as predicted. Finally, the model overestimates the $H$-band surface brightness of the tenuous outer halo beyond $\sim 2$ !" 3 asymmetrically in F110W, where the flux densities on both axes are well matched in the northwest portion of the disk but overpredicted toward the southeast. This suggests that the Terebey et al. (1984) collapse geometry, which we adopted for the for the infalling envelope model and which falls off at large distances as $r^{-1.5}$, may be inappropriate for an evolved tenuous envelope and should be reconsidered in future, more detailed models of this disk system.

Our scattered light model succeeds at reproducing the size, overall brightness level, large-scale surface brightness distribution, colors, and global morphology of GM Aurigae's circumstellar material, including the width of the dusty disk midplane and the presence of light scattered by a tenuous envelope. The photometric fidelity of the model fails to correctly predict some of the small spatial scale features of the disk to high precision. The discrepancies in the scattered light intensity pattern close to the central star are likely due to the combination of our adopted smooth axisymmetric circumstellar geometry along with a simple Henyey \& Greenstein (1941) dust-scattering phase function. Although beyond the scope of our current modeling, a more detailed investigation into the dust-scattering properties will be possible using the combination of high-resolution multiwavelength images (such as our NICMOS data) and resolved polarization data (Potter 2002).

\section{SPECTRAL ENERGY DISTRIBUTION}

We verified the consistency of the scattered light model with a panchromatic $(0.3-2700 \mu \mathrm{m})$ fit to the observed spectral energy distribution (Fig. 9) for the systemic parameters indicated by the scattered light model. For the SED model we used the Monte Carlo radiative equilibrium techniques described by Bjorkman \& Wood (2001) as modified for T Tauri disks (Wood et al. 2002).

The underlying stellar photospheric contribution to the SED was computed from a model stellar atmosphere (Kurucz 1997) with an effective photospheric temperature

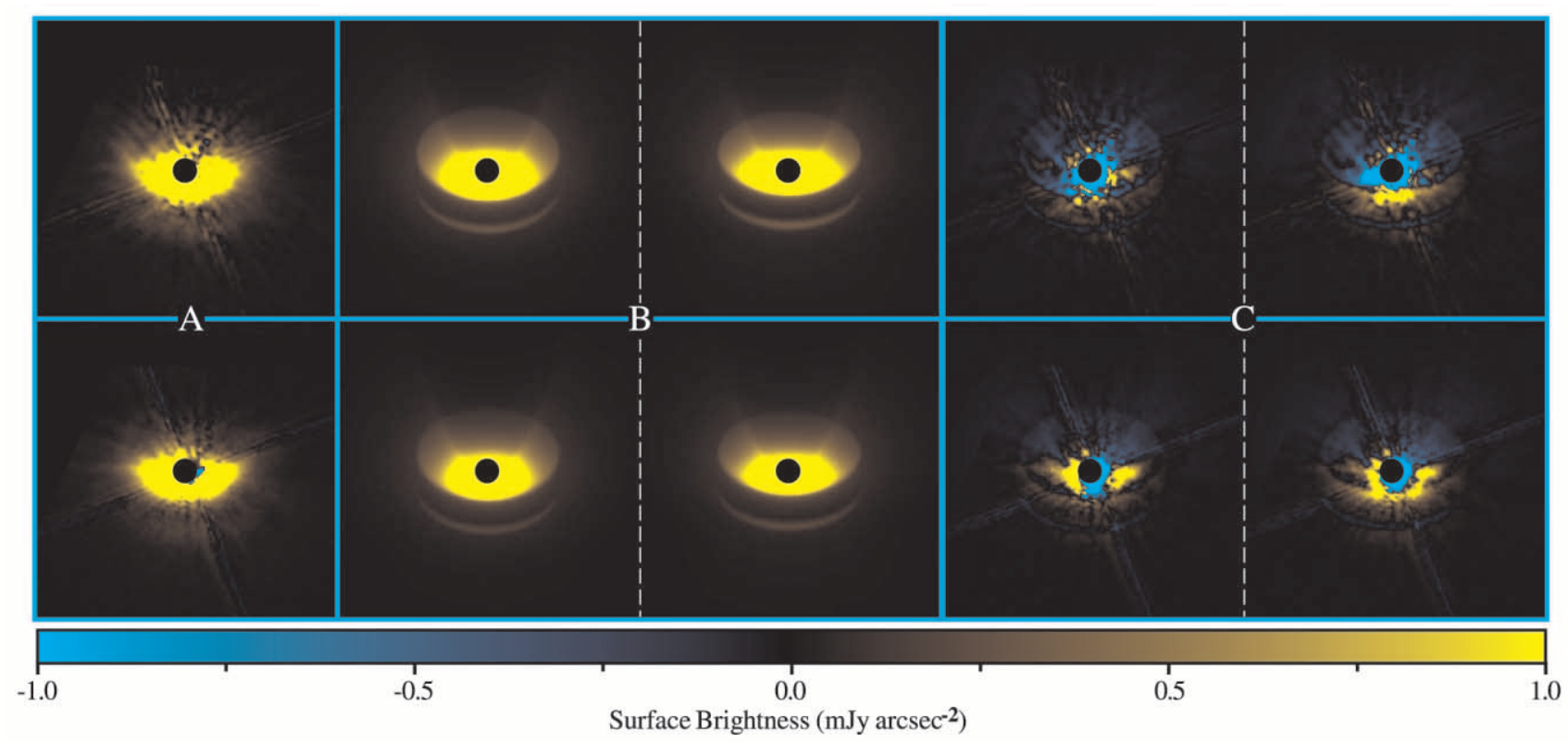

FIG. 7.- (a) Observations; $(b)$ models; $(c)$ difference (observed minus model) images of the GM Auriagae disk, applying the scattered light model discussed in $\S 5$ and 5.1. Top, F160W; bottom, F110W. Models and model subtractions with inclinations of $50^{\circ}$ and $55^{\circ}$ from the plane of the sky are shown to the left and right of the dotted lines, respectively. The images have been oriented to place the disk major axis (PA $=58^{\circ} .5$ on the sky) along the image horizontal. The dynamic range of these linear displays covers surface brightnesses from 0 to $1 \mathrm{mJy} \mathrm{arcsec}{ }^{-2}$ for the observations and models, and -1 to $1 \mathrm{mJy}$ arcsec ${ }^{-2}$ for the difference images. The region near the star is saturated at this stretch but provides a direct comparison with the amplitude of the residuals after subtracting the models in the difference images. The scattered light models were convolved with a 0 ". 1 FWHM Gaussian kernel to facilitate a more direct comparison with the observational data. 


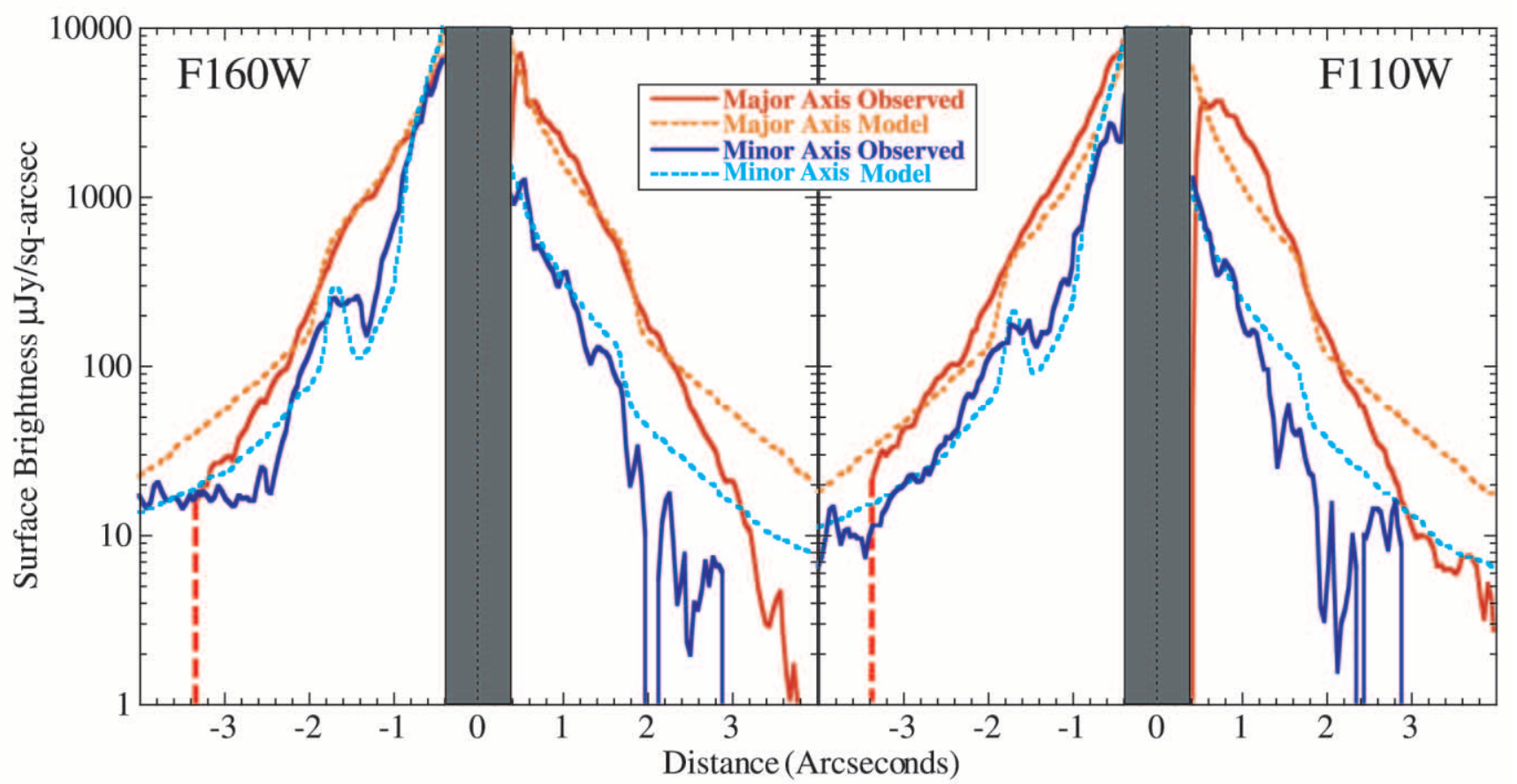

FIG. 8. - Surface brightness profiles of the GM Aurigae disk from the observed and $50^{\circ}$ inclination model images, as shown in Fig. 7 , along the disk major and minor axes. Photometric measurements in both filter passbands were made with extraction apertures 0 ". 3 and 0 ". 4 wide, corresponding to three resolution elements at 1.1 and $1.6 \mu \mathrm{m}$, respectively.

of $4000 \mathrm{~K}$. In doing so we recognized the intrinsically variable nature of GM Aurigae, which is large in photometric amplitude in the UV and diminishing but still significant in the near-IR. For example, the COYOTES 1 survey (Bouvier et al. 1993) reported the $U$-band brightness of GM Aurigae varying between 13.2 and 15.6 mag. Cohen \& Kuhi (1979) found GM Aurigae's $H$-band magnitude to be $8.82 \pm 0.02$, and Kenyon \& Hartmann (1995) reported $H=8.73 \pm 0.16$, the later uncertainty indicative of its intrinsic variation and in agreement with our NICMOS F160W measure of $8.74 \pm 0.02$. These $H$-band measures fall below expectations from the Kurucz model by $20 \%-30 \%$, but are in close agreement with $H=8.578 \pm 0.026$ as determined by 2MASS. The $10 \mu \mathrm{m}$ measure by Cohen (1974) is a $3 \sigma$ upper limit from a nondetection, but the flux density at $12 \mu \mathrm{m}$ was determined by IRAS as $5.05 \pm 0.27 \mathrm{Jy}$. The $10 \mu \mathrm{m}$ measure by Cohen (1974) is a $3 \sigma$ upper limit from a nondetection, but the flux density at $12 \mu \mathrm{m}$ was determined by IRAS as $5.05 \pm 0.27$ Jy (Weaver \& Jones 1992).

In our SED model the dearth of near-IR excess emission is reproduced by introducing an inner cleared region out to a radius of 4 AU. The large gap in the GM Aurigae disk had been inferred from analytic SED models (Koerner et al. 1993; Chiang \& Goldreich 1999) and is confirmed with our detailed SED calculation. More details of the SED calculation, including a dynamical model for disk clearing via the interaction of a planet with the disk (e.g., Lin \& Papaloizou 1979a, 1979b; Artymowicz \& Lubow 1994), will be presented in Rice et al. (2003).

\section{POLAR LOBES}

We find two centrally condensed but diffuse polar "lobes" (indicative of outflow material), lying symmetrically along the minor axis in opposite directions with respect to GM Aurigae (see Fig. $1 c-1 d$ ). Both lobes are $~ 3 " .5-4^{\prime \prime}$ from the central star which, for a disk inclined $50^{\circ}-55^{\circ}$ to the plane of the sky, corresponds to physical distances of $\sim 900 \mathrm{AU}$ from GM Aurigae. The polar lobes are much brighter in $H$ band (by a factor of at least 3 for the northwest lobe) than under the passband of the F110W filter, as shown in Figure $1 c-1 d$. The southeast lobe, however, is poorly detected in F110W in part because of residual artifacts in the PSF subtraction at that azimuthal angle with respect to GM Aurigae, compromising the photometric measurements at that location.

We considered but rejected the conjecture that the polar lobes are optical artifacts in the NICMOS data. Coronagraphic optical artifacts from decentered PSF subtractions (if they should arise) have unequal brightnesses and are spatially (radially) asymmetric with respect to the occulted star. The morphology and inclination of the GM Aurigae disk itself cause a strong brightness anisotropy across the minor axis (see Fig. 6). Yet the polar lobes are seen symmetrically placed with respect to GM Aurigae and have nearly identical flux densities and spatial extent. ${ }^{7}$ The lobes imaged at 1.1 $\mu \mathrm{m}$, however, appear at the same radial locations as in the $1.6 \mu \mathrm{m}$ image, whereas the radial distances of image artifacts

\footnotetext{
${ }^{7}$ The peak flux densities at the photocentric locations of both polar lobes (at \pm 3.8 from GM Aurigae) are $0.14 \mu \mathrm{Jy}$ per pixel measured in the F160W passband. By integrating over a $1 \operatorname{arcsec}^{2}$ area, the F160W surface brightness in the northern (lower) lobe was found to be $15.2 \mu \mathrm{Jy}$ whereas that of the southern lobe is $12.1 \mu \mathrm{Jy}$. The difference might be intrinsic or due to a small (but uncorrectable) calibration artifact in the reference PSF which, upon subtraction, appears as a darker region (as shown in Fig. 1d) partially obscuring the upper lobe. It is unlikely, however, that this artifact causes an underestimation of the flux density of the southern lobe by more than $\sim 20 \%$. Measurement of the surface brightnesses at the locations of the lobes under the F110W filter is more difficult because of the very low intrinsic flux densities in that passband.
} 


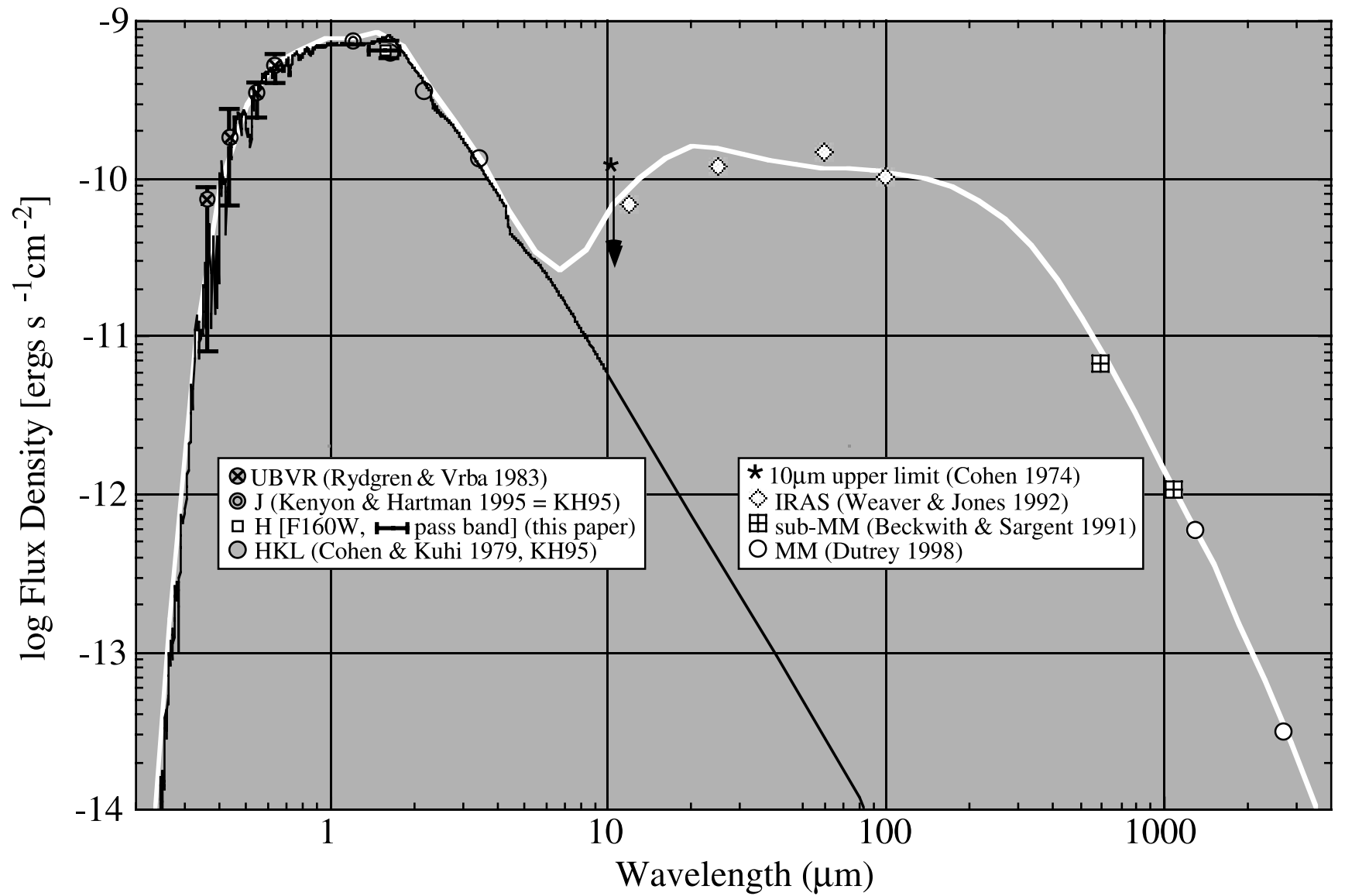

FIG. 9.- Model spectral energy distribution of the GM Aurigae disk (white line), parametrically defined with the systemic parameters found from the scattered light modeling of the NICMOS data (as discussed in $\S 5.1$ ) and fitted to flux density observations in the spectral region spanning $0.3 \mu \mathrm{m}-3 \mathrm{~mm}$. Deviations in the UV through near-IR flux densities as determined from filter photometry (Rydgren \& Vrba 1983), from the photospheric levels predicted by our adopted Kurucz (1997) model atmosphere (black line) and SED fit, are not due to the presence of the disk but rather to intrinsic variability of GM Aurigae (typical peak-to-peak amplitudes indicated by capped gray bars) as discussed in $\S 6$.

(generally) scale linearly with wavelength. Finally we note no such artifacts appear in any of our other NICMOS PSFsubtracted coronagraphic images obtained from more than 80 targets in the NICMOS IDT-EONS disk and companion imaging survey programs.

The disparity in the brightness of the lobes seen in $\mathrm{H}$ band compared with F110W might implicate shocked line emission under the broad F160W filter rather than scattered continuum light. Such emission is expected from ejecta (possibly interacting with the circumstellar medium) from polar mass outflows. Concentrated knots of shocked material are often seen along the polar axes of PMS stars. Magnetohydrodynamic models of such outflows predict relatively large opening angles for mass outflows close to their central stars that become more highly collimated at larger distances (Shu \& Shang 1997).

If the observed brightness of the lobes in $H$ band arises primarily from shocked line emission then one might expect unpolarized radiation from the lobes. Potter (2002) reported imaging the GM Aurigae disk in $H$ band via nulling polarimetric observations with the Gemini telescope's Hokupa'a adaptive optics system. The morphological characteristics of these $H$-band observations of the inner $\sim 2$ ".5 of the disk (the portion of the disk that was detected and imaged via differential polarimetry) are in good agreement with the higher surface brightness regions of the NICMOS images. The lobes, with $H$-band surface brightnesses of $\sim 10 \mu \mathrm{Jy}$ $\operatorname{arcsec}^{-2}$ (as measured with NICMOS), were undetected in the Gemini/Hokupa'a images. However, the instrumental sensitivities were such that the lobes could have been detected only if the intrinsic polarization exceeded $\sim 40 \%$ $50 \%$ (D. Potter 2002, private communication). Hence, the conjecture of unpolarized radiation due to line emission cannot be tested with these polarimetric observations.

A ${ }^{13} \mathrm{CO}(2 \rightarrow 1)$ map of the region around GM Aurigae by Dutrey et al. (1998) shows an emission feature at a distance of $\sim 5$ ". 3 from the peak of the $\mathrm{CO}$ continuum along the rotation axis of the disk. The ${ }^{13} \mathrm{CO}(2 \rightarrow 1)$ feature lies just beyond the location of the NICMOS F160W polar lobe, at the same position angle, further implicating a jetlike outflow phenomenon in GM Aurigae. Such outflows are often episodic, and multiple knots and $\mathrm{HH}$ objects are seen in the collimated outflows of young stars. The placement and orientation of GM Aurigae in the NICMOS coronagraph precluded imaging the disk polar axis beyond $\sim 7^{\prime \prime}$ in both directions from the star. Therefore, speculation of the possibility of an earlier outflow episode can be investigated only by follow-up observations.

\section{BLUE RIBBON}

A broad ( $\sim 3^{\prime \prime}$ wide) and kinked diffuse band of material is seen in the region of GM Aurigae extending 13" toward the northeast from the northeast side of the disk. Near-IR 
light from this "ribbon" is brighter in the F110W band by a factor of 2-3 compared with F160W. This material also appears to brighten, in both bands, as it approaches the GM Aurigae disk. However, this could simply be due to the superposition of this feature with the disk itself. It is not possible to ascertain from these images whether the ribbon is physically contiguous with the disk as opposed to being a foreground (or background) nebulosity. Additionally, the truncation of the field of view to the southwest at a distance of $\sim 4$." 5 from GM Aurigae along the disk major axis precludes investigating whether or not the spatial distribution of this ribbon extends to the southwest side of the disk.

The morphology of the ribbon of material extending from the GM Aurigae disk bears a striking resemblance to that of the nebulosity associated with the class 1 protostar TMC-1 (Terebey et al. 2000). Dust in the form of Pleadies-like reflection nebulosities, likely of interstellar origin, has been found in the close environments of a number of Vega-like stars (Kalas et al. 2002) of spectral types K2 V-B9.5 V. Whether the material apparently extending from the GM Aurigae circumstellar environment results from the disk or is a remnant of the environment from which the disk system formed (or perchance is an unrelated nebulosity) is a subject for future investigation.

\section{SUMMARY}

NICMOS PSF-subtracted coronagraphic observations have spatially resolved the GM Aurigae circumstellar disk from 0 "!35 to $5^{\prime \prime}(\sim 50-700 \mathrm{AU})$ from the star. The disk is inclined by $50^{\circ}-55^{\circ}$ to the plane of the sky. Scattered nearIR light from the disk comes from two regions, a bright " upper" component, which is outwardly flared, and a faint "lower" component seen in the direction of forward scattering "below" the darker (high opacity) midplane of the disk. GM Aurigae is largely unobscured and is bilaterally centered in the upper portion of the disk. Only the upper surface of the lower portion of the disk, unshadowed by the midplane, is seen. The disk very likely has an intrinsic morphology similar to HH 30, HK Tau, and Haro 6-5B, though the lower portion of the GM Aurigae disk is more difficult to detect because of the viewing geometry.

We measure flux densities of 8.0 and $9.3 \mathrm{mJy}( \pm 20 \%)$ at F110W and F160W for the integrated brightness of the disk, excluding the inner 0 ". 35 , and find $L_{\text {disk }} / L_{*}=0.025( \pm 20 \%)$ under the passbands of both filters. The disk brightness declines along the minor axis as $r^{-2.9}$ and $r^{-3.5}$ at F110W and $\mathrm{F} 160 \mathrm{~W}$, respectively, in the forward direction. By modeling the scattered light from a passively heated flared disk with a tenuous outer infall envelope we find the disk mass to be $\sim 0.04 M_{\odot}$.

We find diffuse lobes of likely outflow material at 3".5-4" from GM Aurigae in both directions along the disk minor axis. Though marginally detectable at F110W, these polar lobes are readily apparent at $\mathrm{F} 160 \mathrm{~W}$ with a peak brightness of $\sim 0.14 \mu \mathrm{Jy}$ per camera 2 pixel $\left(\sim 15.2 \mu \mathrm{Jy} \operatorname{arcsec}^{-2}\right)$. We conjecture these features are primarily due to shocked emission from jets of outflow material based upon the disparity in F160W and F110W brightness and the appearance of an adjacent CO emission feature (Fig. 1 of Dutrey et al. 1998) also seen on the polar axis. Additionally, we note the existence of an $\sim 3^{\prime \prime}$ wide band of diffuse blue material, at least $12^{\prime \prime}$ in length, seen to the northeast and spatially adjoining the disk (possibly only in projection). These characteristics are consistent with the general paradigm of disk evolution for an older classical $\mathrm{T}$ Tauri star.

The authors wish to thank the other members of the NICMOS IDT EONS team for their contributions to this investigation. We gratefully acknowledge information provided by D. Potter concerning his polarimetric nulling observations of the GM Aurigae disk. We also wish to thank C. Grady for her insightful and helpful commentary on a number of issues addressed in this paper. This work was supported by NASA grant NAG 5-3042 to the NICMOS IDT. This paper is based on observations with the NASA/ ESA Hubble Space Telescope, obtained at the Space Telescope Science Institute, which is operated by the Association of Universities for Research in Astronomy, Inc., under NASA contract NAS 5-26555. K. W. acknowledges support from a UK PPARC Advanced Fellowship.
Artymowicz, P., \& Lubow, S. H. 1994, ApJ, 421, 651

Aumann, H. H. 1985, PASP, 97, 885

Beckwith, S. V. W., \& Sargent, A. I. 1991, ApJ, 381, 250

Beckwith, S. V. W., Sargent, A. I., Chini, R. S., \& Guesten, R. 1990, AJ, 99 , 924

Bjorkman, J. E., \& Wood, K. 2001, ApJ, 554, 615

Bouvier, J., Cabrit, S., Fernandez, M., Martin, E. L., \& Matthews, J. M. 1993, A\&AS, 101, 485

Bouvier, J., Covino, E., Kovo, O., Martin, E. L., Matthews, J. M.,

Terranegra, L., \& Beck, S. C. 1995, VizieR Online Data Catalog, 329, 90089

Burrows, C. J., et al. 1996, ApJ, 473, 437

Cardelli, J. A., Clayton, G. C., \& Mathis, J. S. 1988, ApJ, 329, L33 .1989, ApJ, 345, 245

Chiang, E. I., \& Goldreich, P. 1997, ApJ, 490, 368 1999, ApJ, 519, 279

Cohen, M. 1974, MNRAS 169, 257

Cohen, M., \& Kuhi, L. V. 1979, ApJS, 41, 743

Cotera, A. S., et al. 2001, ApJ, 556, 958

D’Alessio, P., Calvet, N., Hartmann, L., Lizano, S., \& Canto, J. 1999, ApJ, 527,893

Dutrey, A., Guilloteau, S., Prato, L., Simon, M., Duvert, G., Schuster, K.,

\& Menard, F. 1998, A\&A, 338, L63

Folha, D. F. M., \& Emerson, J. P. 2001, A\&A, 365, 90

Grady, C. A., et al. 2000, ApJ, 544, 895

\section{REFERENCES}

Grady, C. A., et al. 2001, BAAS, 199(6015)

Gullbring, E., Hartmann, L., Briceno, C., \& Calvet, N. 1998, ApJ, 492, 323

Henyey, L. G., \& Greenstein, J. L. 1941, ApJ, 93, 70

Kalas, P., Graham, J. R., Beckwith, S. V. W., Jewitt, D. C., \& Lloyd, J. P. 2002, ApJ, 567, 999

Kenyon, S. J., \& Hartmann, L. 1995, ApJS, 101, 117

Koerner, D. W., Sargent, A. I., \& Beckwith, S. V. W. 1993, Icarus, 106, 2

Koerner, D. W., et al. (NICMOS IDT EONS teams) 1998, BAAS, 193(7314)

Konig, B., Neuhauser, R., \& Stelzer, B. 2001, A\&A, 369, 971

Kurucz, R. L. 1997, The First ISO Workshop on Analytical Spectroscopy (ESA SP-419) (Noordwijk: ESA), 193

Lin, D. N. C., \& Papaloizou, J. 1979a, MNRAS, 186, 799 1979b, MNRAS, 188, 191

Looney, L. W., \& Mundy, L. G. 2000, ApJ, 529, 477

Padgett, D. L., Brandner, W., Stapelfeldt, K. R., Strom, S. E., Terebey, S., \& Koerner, D. 1999, AJ, 117, 1490

Potter, D. 2002, Ph. D. thesis, Univ. Hawaii

Rice, W. M. K., et al. 2003, in preparation

Rydgren, A. E., \& Vrba, F. J. 1983, AJ, 88, 1017

Schneider, G. 2003, in ASP Conf. Ser. 29, Hubble's Science Legacy: Future Optical-Ultraviolet Astronomy from Space, ed. K. R. Sembach, G. D. Illingworth, R. C. Kennicutt, \& C. Blades (San Francisco: ASP), 69

Schneider, G., Becklin, E. E., Smith, B. A., Weinberger, A. J., Silverstone, M., \& Hines, D. C. 2001, AJ, 121, 525 
Schneider, G., \& Silverstone, M. 2002, Proc. SPIE, 4860, in press

Schneider, G., \& Stobie, E. 2002, ASP Conf. Ser. 281, Astronomical Data

Analysis Software and System XI, ed. D. A. Bohlender, D. Durand, \& T. H. Handley (San Francisco: ASP)

Schneider, G., Thompson, R. I., Smith, B. A., \& Terrile, R. J. 1998, Proc. SPIE, 3356, 222

Schultz, A., et al. 2001, NICMOS Instrument Handbook, Version 4.1 (Baltimore: STScI)

Shakura, N. I., \& Sunyaev, R. A. 1973, A\&A, 24, 337

Shu, F. H., \& Shang, H. 1997, in IAU Symp. 182: Herbig-Haro Flows and the Birth of Low-Mass Stars, ed. B. Reipurth \& C. Bertout (Dordrecht: Kluwer), 182, 225

Siess, L., Forestini, M., \& Bertout, C. 1999, A\&A, 342, 480

Simon, M., Dutrey, A., \& Guilloteau, S. 2000, ApJ, 545, 1034

Skrutskie, M. F., Dutkevitch, D., Strom, S. E., Edwards, S., Strom, K. M., \& Shure, M. A. 1990, AJ, 99, 1187

Stapelfeldt, K., et al. (WFPC2 IDT team) 1997, in Science with the VLT Interferometer, ed. F. Paresce (Berlin: Springer), 395

Stapelfeldt, K. R., Krist, J. E., Menard, F., Bouvier, J., Padgett, D. L., \& Burrows, C. J. 1998, ApJ, 502, L65

Stobie, E., Lytle, D., Barg, I., \& Ferro, A. 1998, NICMOS and the VLT: A New Era of High Resolution Near-Infrared Imaging and Spectroscopy, ed. Freudling \& R. Hook (Munich: ESO), 96
Tamura, M., Hough, J. H., Greaves, J. S., Morino, J., Chrysostomou, A., Holland, W. S., \& Momose, M. 1999, ApJ, 525, 832

Terebey, S., Shu, F. H., \& Cassen, P. 1984, ApJ, 286, 529

Terebey, S., Van Buren, D., Matthews, K., \& Padgett, D. L. 2000, AJ, 119, 2341

Weaver, W. B., \& Jones, G. 1992, ApJS, 78, 239

Weinberger, A. J., et al. 2002, ApJ, 566, 409

Weinberger, A. J., Becklin, E. E., Schneider, G., Smith, B. A., Lowrance, P. J., Silverstone, M.D., Zuckerman, B., \& Terrile, R. J. 1999, ApJ, 525, L53

White, R. J., \& Ghez, A. M. 2001, ApJ, 556, 265

White, R. J., Ghez, A. M., Reid, I. N., \& Schultz, G. 1999, ApJ, 520, 811

Whitney, B. A., \& Hartmann, L. 1992, ApJ, 395, 529 1993, ApJ, 402, 605

Wood, K., Kenyon, S. J., Whitney, B. A., \& Turnbull, M. 1998, ApJ, 497, 404

Wood, K., Lada, C. J., Bjorkman, J. E., Kenyon, S. J., Whitney, B. A., \& Wolff, M. J. 2002, ApJ, 567, 1183

Wood, K., Smith, D., Whitney, B., Stassun, K., Kenyon, S. J., Wolff, M. J., \& Bjorkman, K. S. 2001, ApJ, 561, 299 